\title{
To AND or To OR: On Energy-Efficient Distributed Spectrum Sensing with Combined Censoring and Sleeping
}

\author{
Sina Maleki, Geert Leus, Symeon Chatzinotas and Björn Ottersten
}

\begin{abstract}
Distributed spectrum sensing improves the detection reliability of a cognitive radio network but generally comes at the price of a large power consumption. Since cognitive radios are generally low-power sensors with limited batteries, a combined censoring and sleeping scheme is considered as an energy-efficient algorithm for distributed spectrum sensing. Each sensor switches off its sensing module with a specific sleeping rate. When the sensor is on, a censoring policy is employed in order to send the sensing result to the fusion center. The result is only transmitted, if it is deemed to be informative. Hence, the energy consumption of each sensor, including the sensing and transmission energies, is reduced. The underlying sensing parameters are derived by minimizing the maximum average energy consumption per sensor subject to a lower-bound on the global probability of detection and an upper-bound on the global probability of false alarm. We analyze the problem for the OR and the AND rule and provide a performance analysis for a case study based on the IEEE 802.15.4/ZigBee standard. It is shown that the combined censoring and sleeping scheme achieves a significant energy saving compared to the case where no censoring or sleeping is taken into account.
\end{abstract}

Index Terms-Energy-efficiency, combined censoring and sleeping, distributed spectrum sensing, cognitive radio networks.

\section{INTRODUCTION}

Dynamic spectrum access employing cognitive radios has been proposed, in order to opportunistically operate in underutilized portions of the heavily licensed electromagnetic spectrum [1], [2]. Cognitive radios opportunistically share the spectrum, while avoiding any harmful interference to the primary licensed users. One major category of cognitive radios yields the interweave cognitive radios [3]. In this category, cognitive radios employ spectrum sensing to detect the empty bands of the radio spectrum, also known as spectrum holes. Upon detection of such a spectrum hole, cognitive radios dynamically use this empty band. However, as soon as the primary user appears in the corresponding band, cognitive radios have to vacate the band and search for a new spectrum hole. This way, reliable spectrum sensing becomes a key functionality of a cognitive radio network.

G. Leus is with the Faculty of Electrical Engineering, Mathematics and Computer Science, Delft University of Technology, 2628 CD Delft, The Netherlands (e-mail: g.j.t.leus@tudelft.nl). S. Maleki, S. Chatzinotas and B. Ottersten are with Interdisciplinary Centre for Security, Reliability and Trust (SnT), University of Luxembourg, L-2721, Luxembourg (e-mail: \{sina.maleki, symeon.chatzinotas, bjorn.ottersten $\} @$ uni.lu). This work is supported in part by the NWO-STW under the VICI program (project 10382), the National Research Fund, Luxembourg under the projects SeMIGod, SATSENT and the European FP7 Project CoRaSat. Parts of this paper has been presented at ICC 2014, Sydney, Australia [41].
Several algorithms have been investigated in order to perform spectrum sensing. Three of the most common techniques which are considered in the literature are energy detection, cyclostationary feature detection and matched filtering [4]. Matched filter detection is optimal in terms of the detection reliability. However, perfect knowledge of the primary user signal as well as the channel side information is required, which is often not available at cognitive radios. Among the other two techniques, energy detection has the lowest computational and implementation complexity, but is vulnerable to the noise uncertainty. On the other hand, cyclostationary detection is more robust to the noise uncertainty, but is computationally complex, and needs a higher sensing time to deliver the same detection performance as energy detection, when the noise uncertainty is not taken into account. Note that in the presence of a noise uncertainty, energy detection fails to detect the primary user below a specific SNR independent of the number of observation samples [5]. An overview of the state-of-theart in spectrum sensing for cognitive radio is discussed in [6]. Due to its simplicity and better mathematical tractability, here we employ energy detection for spectrum sensing. However, the algorithm discussed in this paper, can also be employed for cyclostationary detection.

The hidden terminal problem and fading effects have been shown to limit the reliability of single-user spectrum sensing. Distributed cooperative detection has therefore been proposed to improve the detection performance of a cognitive radio network [7], [8]. Cognitive sensors sense the spectrum in periodic sensing slots by collecting a number of observation samples. The observation samples are then processed in order to provide useful data for a fusion center (FC), which is responsible for making the final decision about the presence or absence of the primary user. The data which is received by the FC is either soft processed data such as likelihoodratio test (LRT) results, or hard binary decisions, which are made by individual cognitive users based on their observations. This way, two distinctive categories of decision rules at the FC arise which are known as soft and hard fusion rules. We refer the readers to [9] for a comprehensive study of distributed detection with soft and hard fusion rules. Due to its simplicity and higher energy-efficiency, a distributed detection scheme based on hard fusion of local results is considered in this paper. Note that each cognitive radio decides for the presence or absence of the primary user exclusively based on the observations obtained in the current sensing slot, and the FC also only takes the current decisions of the cognitive radios 
into account ignoring all the previous decisions. Further, [8] shows that for energy detection, the detection performance of hard and soft fusion schemes is comparable.

Cognitive radios often consist of low-power sensors and thus energy consumption is another critical issue which should be accounted for, in cognitive radio system design. Each cognitive radio consumes energy on two fronts. First, a cognitive sensor spends some energy on sensing by collecting the observation samples and processing them. Second, some energy is consumed in order to transmit data to the FC. In this paper, we intend to design an energy-efficient spectrum sensing algorithm which reduces both the sensing and the transmission energy, while adhering to the desired detection performance of a cognitive radio system. The desired detection performance is defined by a lower-bound on the probability of detection and an upper-bound on the probability of false alarm. This way, the primary users are protected from the interference induced by the cognitive radios and the spectrum opportunities are utilized efficiently.

As an energy-efficient algorithm for distributed spectrum sensing, in [20], a combined sleeping and censoring scheme is considered, which can be viewed as the predecessor of this paper. A censoring policy ensures that only the informative decisions are transmitted to the FC and non-informative ones are censored. On top of censoring, a sleeping mechanism is proposed where each cognitive radio turns off its sensing module with a probability $\mu$. The network energy consumption is minimized subject to a constraint on the probability of detection and false alarm. This approach is shown to reduce the network energy consumption dramatically. Note that [20] is based on minimizing the network energy consumption. However, in low-power sensor networks, the individual energy consumption of each sensor is a more critical factor. Hence, in this paper, minimizing the maximum average energy consumption per sensor shall be considered as the objective function. Further, [20] only considers the OR rule as the fusion rule, while in this paper, we shall include the AND rule as well.

As mentioned earlier, a combination of sleeping and censoring is also considered in this paper. The goal is to minimize the maximum average energy consumption per sensor subject to a lower-bound on the probability of detection and an upperbound on the probability of false alarm. This way, a great amount of energy is saved on sensing and transmission. The main contributions and results of this paper are listed as follows

- A combined sleeping and censoring scheme is proposed where each sensor turns off its sensing module with probability $\mu$ at each sensing period. In case the sensor is on, then a censoring policy is employed in order to send the decisions to the FC. As mentioned before, the cognitive radios make a decision solely based on the current observations. If the calculated energy is more than an upper threshold, $\lambda_{2}$, then the decision is that the primary user is present. If the calculated energy turns out to be lower than a lower threshold, $\lambda_{1}$, then a decision is sent to the FC indicating the absence of the primary user. Else, no decision is made and nothing is sent to the FC. Afterward, the underlying detection performance indicators including the global probability of false alarm and detection are derived for the OR and the AND rule. The problem is defined so as to minimize the maximum average energy consumption per sensor subject to a lower-bound on the probability of detection and an upper-bound on the probability of false alarm.

Further, it is shown that the optimal average energy consumption per sensor is obtained when the lower threshold is zero $\left(\lambda_{1}=0\right)$ for the OR rule and approaching infinity $\left(\lambda_{1} \rightarrow \infty\right)$ for the AND rule. This way, one of the three underlying arguments of the optimization problem including $\lambda_{1}, \lambda_{2}$ and $\mu$, is relaxed and the problem reduces to a two-dimensional optimization problem. Further, it is shown that on top of reducing the main problem to a two-dimensional problem, using the interactions between $\lambda_{2}$ and $\mu$, the problem can be reduced to a line-search problem over $\mu$.

- Asymptotic properties of the OR and the AND rule are discussed as the probability of the primary user absence (or presence) approaches zero or one. It is shown that the optimal average energy consumption per sensor decreases with the probability of the primary user absence for the OR rule, thus achieving the lower-bound when the primary user is always absent. While the optimal average energy consumption per sensor increases with the probability of the primary user absence for the AND rule, thus achieving the upper-bound when the primary user is always absent.

- Asymptotic properties of the OR and the AND rule are investigated as the signal-to-noise-ratio (SNR) approaches infinity. It is shown that as the SNR approaches infinity, the optimal solution of the underlying problems, determined by the optimal sleeping rate, becomes independent from the value of the probability of the primary user absence (or presence), as well as the sensing and transmission energies, and only depends on the maximum sleeping rate in the feasible set of the underlying optimization problem.

- We show that the algorithms which shall be provided in this paper, are independent from the type of signal, as far as the observations remain conditionally independent among the sensors.

The remainder of the paper is organized as follows. We discuss some related works in Section II. The system model and detection scheme is presented in Section III and the combined sleeping and censoring policies are introduced. In Section IV, we shall formulate and analyze the underlying optimization problem for the OR and AND rules, followed by some notes on the implications of deterministic instead of statistical signal and channel assumptions on the proposed scheme in Section V. The numerical results are depicted in Section VI. We conclude the paper in Section VII and discuss some ideas for future work.

\section{RELATED WORKS}

Distributed detection based on censoring in sensor networks is discussed in [10]-[12]. Two problem formulations are proposed in order to derive the underlying parameters including 
the lower and upper thresholds for censoring. The probability of miss detection is minimized subject to a constraint on the probability of false alarm and a constraint on the total network energy consumption, in a Neyman-Pearson (NP) problem formulation, while the probability of error is minimized subject to a constraint on the total network energy consumption in a Bayesian problem formulation.

Censoring for spectrum sensing in cognitive radios is investigated in [13]-[15]. The paper [13] considers a censoring rule in order to reduce the communication overhead of a cognitive radio network which employs the OR rule at the FC. In [14], analytical expressions for the sensing parameters are given according to a NP setup for both soft and hard fusion schemes, but unlike [10]-[12], no constraint on the energy consumption is taken into account. A fixed-sample size censoring scheme, as well as a combined censoring and sequential sensing scheme are discussed in [15]. In the fixed-sample size censoring, the number of observation samples per sensing period is fixed, while in the combined censoring and sequential scheme, a truncated sequential sensing technique is employed, where the sensors sequentially collect observation samples until they reach a decision about the presence or absence of the primary user. It is shown that censored truncated sequential sensing outperforms fixed-sample size censoring in terms of energy-efficiency for the desired detection performance of the cognitive radio system.

In [16], censoring for a collaborative cyclostationary detection scheme in cognitive radio networks is considered. The proposed cyclostationarity detection scheme is a generalization of [17], where sensors send their test statistics to the FC for a final decision about the presence or absence of the primary user. A similar censoring rule as in [10] and [12] is employed, in order to only transmit the test statistics which are deemed to be informative. It is shown that this way, the communication overhead reduces significantly, while the performance loss is low. One of the key advantages of collaborative cyclostationary detection is its robustness to the noise uncertainty. Employing the cooperative cyclostationary detection approach proposed in [16] as the sensing technique in the combined censoring and sleeping scheme of this paper, gives an even more energyefficient reliable spectrum sensing technique at low SNR.

The mutual information between the state of signal occupancy and the decision state of the FC for a combination of censoring and sleeping is presented in [18]. However, the energy-efficiency of the system is not directly addressed. A sleeping technique is employed in [19] where the sleeping policy is controlled by learning from the past channel observations.

A joint sensing and decision node selection scheme is considered in [21]. The network energy consumption is minimized subject to a detection performance constraint defined as in [20], in order to determine the sensing nodes from a pool of cognitive radios and subsequently the decision nodes from the selected sensing nodes. The decision nodes are the nodes which send their result to the FC. Since the problem is to be solved by integer programming and such problems are in general NP hard, a convex relaxation is proposed in order to solve the problem as a real problem and later on map the solution from $[0,1]$ to $\{0,1\}$.

Finally, we discuss some recent works related to energyefficient spectrum sensing which are not directly related to the setup presented in this paper, but are still valuable to discuss in order to provide an overview about the current state-ofthe-art. A joint clustering and confidence voting technique is proposed in [22]. The sensors only participate in voting if they are confident about their decision. Further, by clustering, the transmission distance and hence the transmission energy is reduced even further. Clustering can also be combined with the proposed technique in this paper to achieve an even higher energy efficiency. The sensing-throughput trade-off is analyzed in [23] considering a detailed analysis of the circuitlevel power consumption including the energy consumed in the power amplifier, ADC and low-noise amplifier. The total circuit energy consumption is minimized subject to a data rate constraint, in order to determine the sensing parameters. Optimization of the network throughput for energy-constrained cognitive radios is considered in [24] and [25]. Further, [26] maximizes the energy efficiency of a cognitive radio network by determining the sensing order, the sensing stopping time and the access transmission power of the cognitive users with dynamic programming. Similar energy-efficient sensing-access strategies are also considered in [27] and [28].

\section{SySTEM MODEL}

We consider a network of $M$ cognitive radios. Each cognitive radio senses the spectrum in periodic time slots by collecting $N$ samples in each sensing slot, and it possibly sends a local decision to the FC exclusively based on the current observations. The final decision is then made at the FC only based on the current decisions, and fed back to the cognitive radios for further action. Due to its low latency and robustness to link failure, a parallel configuration as shown in Fig. 1 is employed for distributed spectrum sensing. Denoting $\mathcal{H}_{0}$ and $\mathcal{H}_{1}$ as the absence and the presence of the primary user, each cognitive radio solves a binary hypothesis testing problem as follows

$$
\begin{aligned}
& \mathcal{H}_{0}: r_{i j}=w_{i j}, i=1, \ldots, N, j=1, \ldots, M \\
& \mathcal{H}_{1}: \quad r_{i j}=h_{i j} s_{i}+w_{i j}, i=1, \ldots, N, j=1, \ldots, M
\end{aligned}
$$

where $r_{i j}$ denotes the $i$-th sample received at the $j$-th cognitive user, $s_{i}$ is the primary user signal, $h_{i j}$ is the channel gain between the primary user and the $j$-th cognitive radio, and $w_{i j}$ is additive white Gaussian noise with zero-mean and variance $\sigma_{w}^{2}$. Two models for $s_{i}$ and $h_{i j}$ are assumed. First, the signal is assumed to be an i.i.d. Gaussian random variable with zero-mean and variance $\sigma_{s}^{2}$ and $h_{i j}$ is constant during each sensing slot, i.e., $\forall i \in 1, \ldots, N: h_{i j}=h_{j}$ which represents a slow-fading channel with Gaussian symbols. An example of such a model can be an OFDM signal which experiences flat-fading (note that OFDM signal can be modeled by zeromean Gaussian distribution [29]). In the second model, the signal amplitude and phase is assumed to be constant, i.e., $\left|s_{i}\right|=s$ and the channel gain $h_{i j}$ is assumed to be an i.i.d. Gaussian random variable with zero-mean and variance $\sigma_{h, j}^{2}$ which represents a fast-fading channel, e.g. a BPSK signal 


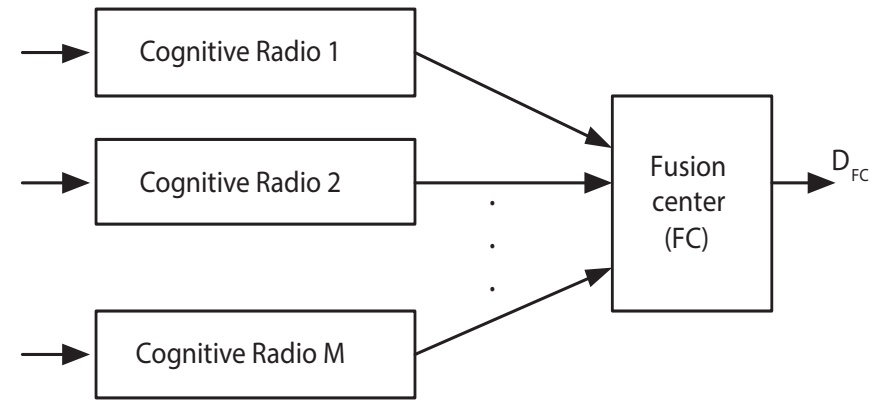

Fig. 1. Distributed spectrum sensing configuration

experiencing a fast-fading channel. This way, denoting $\gamma_{j}$ to be the received average SNR at the $j$-th cognitive radio, $\gamma_{j}=\left|h_{j}\right|^{2} \sigma_{s}^{2} / \sigma_{w}^{2}$ under the first model and $\gamma_{j}=\sigma_{h, j}^{2} s^{2} / \sigma_{w}^{2}$ under the second model. Cognitive radios are not able to estimate the instantaneous channel gain, because the primary user training sequence is generally not available at the secondary users, and further, synchronization with the primary user signal is very difficult as the cognitive radios are not aware of the presence or absence of the primary user at a specific time. However, the received average SNR can be estimated and thus, throughout this paper, we focus on the average received SNR instead of the instantaneous one. We assume that the expected SNR in case of primary user presence is time-invariant during the cognitive radio activity, and can be obtained either through radio environment mapping (REM) [30], or estimated in the beginning of the process [31]. Note that under both models, the received signal remains an i.i.d. zero-mean Gaussian random process with some variance, namely $\sigma_{j}^{2}=\left|h_{j}\right|^{2} \sigma_{s}^{2}+\sigma_{w}^{2}$ for the former model and $\sigma_{j}^{2}=s^{2} \sigma_{h, j}^{2}+\sigma_{w}^{2}$ for the latter model. Therefore, the analyses which are given in the following sections are valid for both models. Furthermore, $h_{i j} s_{i}$ and $w_{i j}$ are assumed statistically independent. Similar signal modeling is frequently used in cognitive radio literature, e.g. [32]-[34]. We shall discuss the implications of a deterministic scenario on our scheme in Section V.

Each cognitive radio accumulates the energy of $N$ samples by employing an energy detector. Considering the adopted system model in this paper, the energy detector is equivalent to the optimal log-likelihood-ratio (LLR) detector [35]. The received energy collected over the $N$ observation samples at the $j$-th radio is given by

$$
\mathcal{E}_{j}=\sum_{i=1}^{N} \frac{\left|r_{i j}\right|^{2}}{\sigma_{w}^{2}} .
$$

A censoring policy is then employed at each radio where the local decisions are sent to the FC, only if they are in a specific information region. The censoring policy is defined by the censoring thresholds $\lambda_{1}$ and $\lambda_{2}$, and the range $\lambda_{1}<\mathcal{E}_{j}<\lambda_{2}$ is called the censoring region. This way, the local censoring decision rule at the $j$-th cognitive radio is given by

$$
\left\{\begin{array}{lr}
\text { send } 1 \text {, declaring } \mathcal{H}_{1} & \text { if } \mathcal{E}_{j} \geq \lambda_{2}, \\
\text { no decision } & \text { if } \lambda_{1}<\mathcal{E}_{j}<\lambda_{2}, \\
\text { send } 0 \text {, declaring } \mathcal{H}_{0} & \text { if } \mathcal{E}_{j} \leq \lambda_{1}
\end{array}\right.
$$

Since the received samples are i.i.d. Gaussian with zeromean, $\mathcal{E}_{j}$ follows a central chi-square distribution with $2 N$ degrees of freedom under $\mathcal{H}_{0}$ and $\mathcal{H}_{1}$. Therefore, the local probabilities of false alarm and detection, respectively become

$$
P_{f, j}=\operatorname{Pr}\left(\mathcal{E}_{j} \geq \lambda_{2} \mid \mathcal{H}_{0}\right)=\frac{\Gamma\left(N, \frac{\lambda_{2}}{2}\right)}{\Gamma(N)},
$$

and

$$
P_{d, j}=\operatorname{Pr}\left(\mathcal{E}_{j} \geq \lambda_{2} \mid \mathcal{H}_{1}\right)=\frac{\Gamma\left(N, \frac{\lambda_{2}}{2\left(1+\gamma_{j}\right)}\right)}{\Gamma(N)},
$$

where $\operatorname{Pr}$ denotes probability and $\Gamma(a, x)$ is the incomplete gamma function given by $\Gamma(a, x)=\int_{x}^{\infty} t^{a-1} e^{-t} d t$, with $\Gamma(a, 0)=\Gamma(a)$. In order to achieve a higher energy saving, on top of censoring, a sleeping policy is applied. Each sensor turns off its sensing module randomly with a sleeping rate denoted by $\mu$. This process can be implemented in each sensor by ordering the sleep/awake slots such that the number of sleep slots is $\mu$ times the total number of sensing slots. The ordering should be totally random and independent from other sensors. Denoting by $C_{s}$ and $C_{t}$ the energy consumed by the $j$-th radio in sensing per sample and transmission per bit, respectively, our cost function is given by the average energy consumption per sensor as follows

$$
C_{j}=(1-\mu)\left(N C_{s}+C_{t}\left(1-\rho_{j}\right)\right)
$$

where $\rho_{j}=\operatorname{Pr}\left(\lambda_{1}<\mathcal{E}_{i}<\lambda_{2}\right)$ represents the censoring rate. It is assumed that $\mu \neq 0$ and $\rho_{j} \neq 0$. Note that in this paper, the transmission and sensing energy of the sensors is assumed to be the same. In practice, this assumption holds when the sensors employ similar radio transceivers. Further, the sensors consume energy during wake-up and idle periods as well. However, these energy sinks are much smaller than the amount of energy spent on the transmission and sensing procedures. Therefore, in this paper, the energy consumption model includes only the transmission and sensing energies, which can be considered as a good approximation of the complete energy consumption model and provides a good platform for further analytical work. Defining $\pi_{0}=\operatorname{Pr}\left(\mathcal{H}_{0}\right)$, $\pi_{1}=\operatorname{Pr}\left(H_{1}\right), \delta_{0, j}=\operatorname{Pr}\left(\lambda_{1}<\mathcal{E}_{j}<\lambda_{2} \mid \mathcal{H}_{0}\right)$ and $\delta_{1, j}=\operatorname{Pr}\left(\lambda_{1}<\mathcal{E}_{j}<\lambda_{2} \mid \mathcal{H}_{1}\right)$, the censoring rate is given by

$$
\begin{gathered}
\rho_{j}=\pi_{0} \operatorname{Pr}\left(\lambda_{1}<\mathcal{E}_{j}<\lambda_{2} \mid \mathcal{H}_{0}\right) \\
+\pi_{1} \operatorname{Pr}\left(\lambda_{1}<\mathcal{E}_{j}<\lambda_{2} \mid \mathcal{H}_{1}\right) \\
=\pi_{0} \delta_{0, j}+\pi_{1} \delta_{1, j},
\end{gathered}
$$

where $\delta_{0, j}$ and $\delta_{1, j}$ are obtained as follows

$$
\begin{gathered}
\delta_{0, j}=\operatorname{Pr}\left(\lambda_{1}<\mathcal{E}_{j}<\lambda_{2} \mid \mathcal{H}_{0}\right) \\
=\frac{\Gamma\left(N, \frac{\lambda_{1}}{2}\right)}{\Gamma(N)}-\frac{\Gamma\left(N, \frac{\lambda_{2}}{2}\right)}{\Gamma(N)}, \\
\delta_{1, j} \quad=\operatorname{Pr}\left(\lambda_{1}<\mathcal{E}_{j}<\lambda_{2} \mid \mathcal{H}_{1}\right) \\
=\frac{\Gamma\left(N, \frac{\lambda_{1}}{2\left(1+\gamma_{j}\right)}\right)}{\Gamma(N)}-\frac{\Gamma\left(N, \frac{\lambda_{2}}{2\left(1+\gamma_{j}\right)}\right)}{\Gamma(N)} .
\end{gathered}
$$

Note that $P_{f, 1}=P_{f, 2}=\cdots=P_{f, M}=P_{f}$ and $\delta_{0,1}=$ $\delta_{0,2}=\cdots=\delta_{0, M}=\delta_{0}$ due to the fixed noise variance over the different cognitive radios. However, this is not true for 
$P_{d, j}$ and $\delta_{1, j}$. So from now on, we will simply use $P_{f}$ and $\delta_{0}$. The FC employes either the OR or the AND rule in order to make the final decision about the presence or absence of the primary user. This means that the FC shall decide for the presence of the primary user, if at least one cognitive radio votes in favor of $\mathcal{H}_{1}$ for the OR rule, and for the absence of the primary user if at least one cognitive radio votes in favor of $\mathcal{H}_{0}$ for the AND rule. In the following section, we shall derive the global probabilities of false alarm and detection for both rules. Further, we provide the corresponding analyses and problem formulations in detail for each rule.

\section{Analysis AND PRoblem Formulation}

As mentioned earlier, the cognitive radio should avoid harmful interference to the primary user. A constraint on the global probability of detection denoted by $Q_{\mathrm{D}}$ is thus dictated to satisfy this requirement. Further, to increase the cognitive network throughput, correct detection of a spectrum hole is very important and thus the probability of false alarm denoted by $Q_{\mathrm{F}}$ shall be designed to be as low as possible. Ideally, we would like $Q_{\mathrm{D}}=1$ and $Q_{\mathrm{F}}=0$. However, in real systems, such an ideal case is impossible. Therefore, we define an upper-bound denoted by $\alpha$ for $Q_{\mathrm{F}}$ and a lower-bound denoted by $\beta$ for $Q_{\mathrm{D}}$. Our goal is then to design the system parameters including $\lambda_{1}, \lambda_{2}$ and $\mu$ by minimizing the maximum average energy consumption per sensor subject to a constraint on the probabilities of false alarm and detection, as follows

$$
\begin{aligned}
& \min _{\mu, \lambda_{1}, \lambda_{2}} \max _{j} C_{j} \\
& \text { s.t. } Q_{\mathrm{F}} \leq \alpha, Q_{\mathrm{D}} \geq \beta .
\end{aligned}
$$

Depending on the prior knowledge about the a priori probabilities, $\pi_{0}$ and $\pi_{1}$, we consider two different cases: a) a blind setup, where $\pi_{0}$ and $\pi_{1}$ are unknown, but $\pi_{1}$ is known to be much smaller than $\pi_{0}$, reflecting channel under-utilization. And b) a knowledge-aided setup where $\pi_{0}$ and $\pi_{1}$ are known. As shall be shown later, the blind setup is a special case of the knowledge-aided setup, where $\pi_{1} \rightarrow 0$. Note that the blind setup could also be defined as the case where $\pi_{1}>\pi_{0}$, however this scenario is not of practical interest in cognitive radio applications. Therefore, without loss of generality, here, we focus on the analysis and problem formulation for the knowledge-aided setup.

In this section, first, we derive $Q_{\mathrm{F}}$ and $Q_{D}$, followed by an analysis of (14) for the OR rule in Section IV-A, and then we follow the same rationale for the AND rule in Section IV-B. Finally, we discuss the asymptotic properties of combined censoring and sleeping for the OR and the AND rules as the SNR approaches infinity in Section IV-C.

\section{A. OR rule}

In this subsection, the FC employs the OR rule in order to make the final decision. Denoting $D_{\mathrm{FC}}$ to be the decision made at the FC, the OR rule means that $D_{\mathrm{FC}}=1$ if at least one cognitive radio sends a 1 , else $D_{\mathrm{FC}}=0$. This way, the global probability of false alarm $Q_{\mathrm{F}, \mathrm{OR}}$ for the OR rule is obtained by

$$
\begin{aligned}
Q_{\mathrm{F}, \mathrm{OR}} & =\operatorname{Pr}\left(D_{\mathrm{FC}}=1 \mid \mathcal{H}_{0}\right) \\
& =1-\operatorname{Pr}\left(D_{\mathrm{FC}}=0 \mid \mathcal{H}_{0}\right) \\
& =1-\left[1-(1-\mu) P_{f}\right]^{M},
\end{aligned}
$$

where $P_{f}$ is given by (4). This can be easily explained by the OR rule based global probability of false alarm when considering $(1-\mu) P_{f}$ to be the local probability of false alarm including the sleeping policy. Note that the false alarm and detection rates are independent from censoring, because if a sensor does not transmit a result to the FC, the FC assumes that the decision of this sensor is zero.

The global probability of detection $Q_{\mathrm{D}, \mathrm{OR}}$ for the OR rule can be derived in a similar way, and results in

$$
\begin{array}{cc}
Q_{\mathrm{D}, \mathrm{OR}} & =\operatorname{Pr}\left(D_{\mathrm{FC}}=1 \mid \mathcal{H}_{1}\right) \\
& =1-\operatorname{Pr}\left(D_{\mathrm{FC}}=0 \mid \mathcal{H}_{1}\right) \\
= & 1-\prod_{j=1}^{M}\left[1-(1-\mu) P_{d, j}\right],
\end{array}
$$

where $P_{d, j}$ is given by (5). This also can be explained by the OR rule based global probability of detection when considering $(1-\mu) P_{d, j}$ to be the local probability of detection including the sleeping policy.

To analyze (14) for the OR rule, it is more convenient to rewrite it in the following format

$$
\begin{aligned}
& \min _{\mu, \lambda_{1}, \lambda_{2}} \max _{j}(1-\mu)\left[N C_{s}+C_{t}\left(1-\rho_{j}\right)\right] \\
& \text { s.t. } 1-\left[1-(1-\mu) P_{f}\right]^{M} \leq \alpha \\
& 1-\prod_{j=1}^{M}\left[1-(1-\mu) P_{d, j}\right] \geq \beta
\end{aligned}
$$

Since for the OR rule, the FC only decides on the presence of the primary user by receiving $1 \mathrm{~s}$, sending 0 s is not optimal in terms of energy efficiency. Therefore, $\lambda_{1}=0$ is the optimal solution to (21). Using this result, we can relax one of the arguments of the problem. When $\lambda_{1}=0$, we obtain

$$
1-\delta_{0}=P_{f}, 1-\delta_{1, j}=P_{d, j} .
$$

Hence, (21) is given by

$$
\begin{aligned}
& \min _{\mu, \lambda_{2}} \max _{j}(1-\mu)\left[N C_{s}+C_{t}\left(\pi_{0} P_{f}+\pi_{1} P_{d, j}\right)\right] \\
& \text { s.t. } 1-\left[1-(1-\mu) P_{f}\right]^{M} \leq \alpha, \\
& 1-\prod_{j=1}^{M}\left[1-(1-\mu) P_{d, j}\right] \geq \beta .
\end{aligned}
$$

Since $\mu, \lambda_{2}$ (and thus $P_{f}$ ), $C_{s}$, and $C_{t}$ are the same among the sensors, the maximum average energy consumption belongs to the sensor with maximum probability of detection. This in turn is acheived by the user with the maximum SNR. Therefore, denoting $j^{*}$ as the user with maximum average energy consumption, we obtain $j^{*}=\max _{j}\left(\gamma_{j}\right)$. Note that since the expected SNR is assumed to be time-invariant, $j^{*}$ is also time-invariant. From (21), we can see that if the values $C_{s}$ and $C_{t}$ are different among the sensors, determining the user with the maximum average energy consumption becomes dependent on the specific values of $\mu$ and $\rho$. In this situation, 
solving the min-max problem becomes mathematically intractable. Therefore, we need to apply a seemingly sub-optimal approach called person-by-person optimization in each individual sensor by minimizing the average energy consumption, and then determine $\mu$ and $\rho$ from the sensor which delivers the maximum minimum average energy consumption among the sensors. Considering $j=j^{*}$, for a fixed value of $\mu=\mu^{*}$, (24) reduces to the following problem

$$
\begin{aligned}
& \min _{P_{f}}\left(1-\mu^{*}\right)\left[N C_{s}+C_{t}\left(\pi_{0} P_{f}+\pi_{1} P_{d, j^{*}}\right)\right] \\
& \text { s.t. } P_{f} \leq \frac{1-(1-\alpha)^{1 / M}}{\left(1-\mu^{*}\right)}, \\
& 1-\prod_{j=1}^{M}\left[1-\left(1-\mu^{*}\right) P_{d, j}\right] \geq \beta,
\end{aligned}
$$

where we exchanged $\lambda_{2}$ in (24) with $P_{f}$, because $\lambda_{2}$ is a one-to-one function of $P_{f}$ [36]. Defining $F\left(\lambda_{2}\right)=\frac{\Gamma\left(N, \frac{\lambda_{2}}{2}\right)}{\Gamma(N)}$, we can write $P_{d, j^{*}}$ as $P_{d, j^{*}}=F\left(\lambda_{2} /\left(1+\gamma_{j^{*}}\right)\right)$. Calculating the derivative of $C_{j^{*}}$ with respect to $P_{f}$, we find that $\frac{\partial C_{j^{*}}}{\partial P_{f}}=$ $\left(1-\mu^{*}\right) \frac{\partial\left[C_{t}\left(\pi_{0} P_{f}+\pi_{1} P_{d, j^{*}}\right)\right]}{\partial P_{f}}=\left(1-\mu^{*}\right)\left[C_{t} \pi_{0}+\frac{\partial P_{d, j^{*}}}{\partial P_{f}}\right] \geq 0$ where we used the fact that $\frac{\partial P_{d, j^{*}}}{\partial P_{f}} \geq 0$. This way, minimizing $P_{f}$ leads to minimizing $P_{d, j^{*}}$ and thus minimizing the objective function in (26). Therefore we can write (26) as follows

$$
\begin{aligned}
& \min _{P_{f}} P_{f} \\
& \text { s.t. } P_{f} \leq \frac{1-(1-\alpha)^{1 / M}}{\left(1-\mu^{*}\right)}, \\
& 1-\prod_{j=1}^{M}\left[1-\left(1-\mu^{*}\right) P_{d, j}\right] \geq \beta .
\end{aligned}
$$

Looking at (28) we can find that

$$
F\left(G^{-1}\left(\mu^{*}, \beta\right)\right) \leq P_{f} \leq \alpha^{\prime} /\left(1-\mu^{*}\right),
$$

where $G\left(\mu, \lambda_{2}\right)=Q_{\mathrm{D}, \mathrm{OR}}=1-\prod_{j=1}^{M}\left[1-(1-\mu) P_{d, j}\right]$, $\alpha^{\prime}=1-(1-\alpha)^{1 / M}$, and $G^{-1}(\mu, \beta)$ is defined over the second argument of $G(\mu, \beta)$. Thus, we find that for every $\mu^{*}$ for which $F\left(G^{-1}\left(\mu^{*}, \beta\right)\right) \leq \alpha^{\prime} /\left(1-\mu^{*}\right)$, the solution to (28) is given by $P_{f}^{*}=F\left(G^{-1}\left(\mu^{*}, \beta\right)\right)$. Therefore, our optimization problem reduces to the following line search problem which we solve by exhaustive search over $\mu$,

$$
\begin{gathered}
\min _{\mu}(1-\mu)\left[N C_{s}+C_{t}\left(\pi_{0} F\left(G^{-1}(\mu, \beta)\right)\right.\right. \\
\left.\left.+\pi_{1} F\left(G^{-1}(\mu, \beta) /\left(1+\gamma_{j^{*}}\right)\right)\right)\right] .
\end{gathered}
$$

Looking carefully at (31), we find that we can use the same optimization problem for the blind setup by considering $\pi_{0}=1\left(\pi_{1}=0\right)$. In other words, the blind setup is just a special case of the knowledge-aided setup. This is the approach that we will adopt in the simulations for both setups. Further, the following theorem leads to some results regarding the asymptotic properties of (31) when $\pi_{0}$ approaches zero or one.

Theorem 1: If $\pi_{0}$ increases, then the optimal $C_{j}$ decreases for the OR rule.
Proof: The proof is provided in Appendix A.

Two immediate corollaries of Theorem 1 which describe the asymptotic properties of (31) are as follows:

Corollary 1: The lower-bound on the optimal average energy consumption per sensor in (31) is obtained for $\pi_{0}=1$ or when the primary user is always absent.

Corollary 2: The upper-bound on the optimal average energy consumption per sensor in (31) is obtained for $\pi_{0}=0$ or when the primary user is always present.

\section{B. AND Rule}

Here, we analyze the performance of the combined sleeping and censoring for the AND rule. As in Section IV-A, we provide the analysis for the knowledge-aided case. The analysis for the blind problem formulation is then straightforward. According to the AND rule, $D_{\mathrm{FC}}=0$, if at least one cognitive radio reports a zero, else $D_{\mathrm{FC}}=1$. Note that for the AND rule, if the FC receives no decision from the $j$-th cognitive user, it automatically considers this decision to be 1 . This way, the global probabilities of false alarm and detection are obtained as follows

$$
\begin{array}{cc}
Q_{\mathrm{F}, \mathrm{AND}} & =\operatorname{Pr}\left(D_{\mathrm{FC}}=1 \mid \mathcal{H}_{0}\right) \\
= & {\left[\mu+(1-\mu)\left(\delta_{0}+P_{f}\right)\right]^{M}} \\
= & {\left[1-(1-\mu)\left(1-\delta_{0}-P_{f}\right)\right]^{M},} \\
Q_{\mathrm{D}, \mathrm{AND}} & =\operatorname{Pr}\left(D_{\mathrm{FC}}=1 \mid \mathcal{H}_{1}\right) \\
=\prod_{j=1}^{M}\left[\mu+(1-\mu)\left(\delta_{1, j}+P_{d, j}\right)\right] \\
=\prod_{j=1}^{M}\left[1-(1-\mu)\left(1-\delta_{1, j}-P_{d, j}\right)\right] .
\end{array}
$$

These derivations can be easily explained by the AND rule based global probabilities of false alarm and detection when considering $1-\left[(1-\mu)\left(1-\delta_{0}-P_{f}\right)\right]$ and $1-\left[(1-\mu)\left(1-\delta_{1, j}-\right.\right.$ $\left.P_{d, j}\right)$ to be the local probabilities of false alarm and detection including the sleeping and censoring policies, respectively. Note that for the AND rule, the FC considers any result except 0 as 1. Therefore, from the FC viewpoint, a false alarm (or detection) at the $j$-th cognitive radio occurs if the received result is not 0 when the primary user is absent (or present). This happens if the sensor goes to sleep mode at a specific sensing time, or if awake, the sensor either censors the result or sends a 1 to the FC. That is why for the AND rule, the censoring rate plays a role in the global probabilities of false alarm and detection which is not the case for the OR rule.

We define our problem in order to find the underlying arguments $\left(\lambda_{1}, \lambda_{2}, \mu\right)$, so as to minimize the maximum average energy consumption per sensor subject to a constraint on the probabilities of false alarm and detection. As in the previous scenario, the constraints on the probabilities of false alarm and detection are defined by an upper-bound $\alpha$ and a lower-bound $\beta$, respectively. This way, the problem is written as follows

$$
\begin{aligned}
& \min _{\mu, \lambda_{1}, \lambda_{2}} \max _{j} C_{j} \\
& \text { s.t. } Q_{\mathrm{F}, \mathrm{AND}} \leq \alpha, Q_{\mathrm{D}, \mathrm{AND}} \geq \beta .
\end{aligned}
$$


Since the FC decides for $\mathcal{H}_{0}$ only by receiving zeros, the optimal solution of (39) is attained by $\lambda_{2} \rightarrow \infty$. This way, the global probabilities of false alarm and detection reduce to

$$
\begin{gathered}
Q_{\mathrm{F}, \mathrm{AND}}=\left[1-(1-\mu)\left(1-\delta_{0}\right)\right]^{M}, \\
Q_{\mathrm{D}, \mathrm{AND}}=\prod_{j=1}^{M}\left[1-(1-\mu)\left(1-\delta_{1, j}\right)\right] .
\end{gathered}
$$

Inserting (40) and (41) in (39) and relaxing $\lambda_{2}$ using the fact that $\lambda_{2} \rightarrow \infty$ is optimal, we obtain

$$
\begin{aligned}
& \min _{\mu, \lambda_{1}} \max _{j}(1-\mu)\left(N C_{s}+C_{t}\left(1-\rho_{j}\right)\right) \\
& \text { s.t. }\left[1-(1-\mu)\left(1-\delta_{0}\right)\right]^{M} \leq \alpha, \\
& \prod_{j=1}^{M}\left[1-(1-\mu)\left(1-\delta_{1, j}\right)\right] \geq \beta,
\end{aligned}
$$

where $\rho_{j}=\pi_{0} \delta_{0}+\pi_{1} \delta_{1, j}$. Since there is a one-to-one relation between $\lambda_{1}$ and $\delta_{0}$, we can rewrite (42) as follows

$$
\begin{aligned}
& \min _{\mu, \delta_{0}} \max _{j}(1-\mu)\left(N C_{s}+C_{t}\left(1-\pi_{0} \delta_{0}-\pi_{1} \delta_{1, j}\right)\right) \\
& \text { s.t. }\left[1-(1-\mu)\left(1-\delta_{0}\right)\right]^{M} \leq \alpha, \\
& \prod_{j=1}^{M}\left[1-(1-\mu)\left(1-\delta_{1, j}\right)\right] \geq \beta,
\end{aligned}
$$

Since all the parameters in $C_{j}$ are the same among the sensors except $\delta_{1, j}$, unlike the OR rule, the user with the minimum SNR consumes the maximum amount of energy in average. Therefore, $j^{*}=\min _{j} \gamma_{j}$. As in the OR rule, if $C_{s}$ and $C_{t}$ become different among the sensors, a similar person-byperson optimization can be applied. Considering $j=j^{*}$, for a given $\mu=\mu^{*}$, (44) becomes

$$
\begin{aligned}
& \min _{\delta_{0}}\left(1-\mu^{*}\right)\left(N C_{s}+C_{t}\left(1-\pi_{0} \delta_{0}-\pi_{1} \delta_{1, j^{*}}\right)\right) \\
& \text { s.t. }\left[1-\left(1-\mu^{*}\right)\left(1-\delta_{0}\right)\right]^{M} \leq \alpha, \\
& \prod_{j=1}^{M}\left[1-\left(1-\mu^{*}\right)\left(1-\delta_{1, j}\right)\right] \geq \beta .
\end{aligned}
$$

Since $\delta_{1, j^{*}}$ is a monotone increasing function of $\delta_{0}$, the optimal solution of (46) is obtained by solving the following problem

$$
\begin{aligned}
& \max _{\delta_{0}} \delta_{0} \\
& \text { s.t. }\left[1-\left(1-\mu^{*}\right)\left(1-\delta_{0}\right)\right]^{M} \leq \alpha, \\
& \prod_{j=1}^{M}\left[1-\left(1-\mu^{*}\right)\left(1-\delta_{1, j}\right)\right] \geq \beta .
\end{aligned}
$$

Therefore, if the feasible set of (48) is not empty, then the maximum $\delta_{0}$ in this feasible set determines the optimal $\delta_{0}$. From the first constraint in (48), we find $\delta_{0} \leq 1-\frac{1-\alpha^{1 / M}}{1-\mu^{*}}$. Assuming $Q_{\mathrm{D}, \mathrm{AND}}=G\left(\mu, \delta_{0}\right)$, we have $\frac{\partial G\left(\mu, \delta_{0}\right)}{\partial \delta_{0}}=$ $\frac{\partial G\left(\mu, \delta_{0}\right)}{\partial \delta_{1, j}} \frac{\partial \delta_{1, j}}{\partial \delta_{0}} \geq 0$, where we used the fact that $\frac{\partial G\left(\mu, \delta_{0}\right)}{\partial \delta_{1, j}} \geq 0$.
This way, from the second constraint in (48), we obtain $\delta_{0} \geq$ $G^{-1}\left(\mu^{*}, \beta\right)$, where the inverse function is defined over the second argument in $G\left(\mu, \delta_{0}\right)$. Based on this discussion, (48) reduces to $\max _{\delta_{0}} \delta_{0}$ subject to $G^{-1}\left(\mu^{*}, \beta\right) \leq \delta_{0} \leq 1-\frac{1-\alpha^{1 / M}}{1-\mu^{*}}$. Therefore, the optimal $\delta_{0}$ is obtained by $\delta_{0}=1-\frac{1-\alpha^{1 / M}}{1-\mu^{*}}$. Inserting the optimal $\delta_{0}$ for a given $\mu$ in (44), we obtain the following line search problem in order to determine the optimal $\mu$ and consequently $\delta_{0}$ and $\lambda_{1}$ which is solved by exhaustive search.

$$
\begin{aligned}
\min _{\mu}(1-\mu)\left[N C_{s}\right. & +C_{t}\left(1-\pi_{0}\left(1-\frac{1-\alpha^{1 / M}}{1-\mu}\right)\right. \\
& \left.\left.-\pi_{1} F_{j^{*}, \mathrm{AND}}\left(1-\frac{1-\alpha^{1 / M}}{1-\mu}\right)\right)\right]
\end{aligned}
$$

where $F_{j^{*}, \mathrm{AND}}\left(\delta_{0}\right)=\delta_{1, j^{*}}\left(\delta_{0}\right)=F\left(F^{-1}\left(\delta_{0}\right) /\left(1+\gamma_{j^{*}}\right)\right)$. In search for the optimal $\mu$, we should note that $\mu \leq \alpha^{1 / M}$ which comes from the fact that $1-\frac{1-\alpha^{1 / M}}{1-\mu} \geq 0$ and also $G\left(\mu, 1-\frac{1-\alpha^{1 / M}}{1-\mu}\right) \geq \beta$. Further, the following theorem leads to some results regarding the asymptotic properties of (50) when $\pi_{0}$ approaches zero or one.

Theorem 2: If $\pi_{0}$ decreases, the optimal $C_{j}$ decreases for the AND rule.

Proof: The proof is provided in Appendix B.

Two immediate corollaries of Theorem 2 which describe the asymptotic properties of (50) are as follows:

Corollary 3: The lower-bound on the optimal average energy consumption per sensor in (50) is obtained for $\pi_{0}=0$ or when the primary user is always present.

Corollary 4: The upper-bound on the optimal average energy consumption per sensor in (50) is obtained for $\pi_{0}=1$ or when the primary user is always absent.

We note that asymptotic properties of the AND rule are opposite to those of the OR rule in Corollaries 1 and 2 . These corollaries as well as the ones in Section IV-A provide the achievable performance boundaries for the combined censoring and sleeping scheme for the OR and the AND rule. They are particularly important in situations where the exact probability of the primary user absence or presence is not known, such as in the blind setup defined in this paper, where we know that $\pi_{0} \gg \pi_{1}$. In such situations, depending on the value of $\pi_{0}$, we can use either the lower or the upper bound. For example, as shall be shown in Section VI-A, in case of the blind setup, we use the lower-bound on the optimal average energy consumption per sensor in (31) for the OR rule, and the upper-bound in (50) for the AND rule, which are corresponding to Corollaries 1 and 4, respectively.

\section{Asymptotic properties of the OR and AND rules when $\gamma_{j} \rightarrow$ $\infty$}

The following theorem which is proved in Appendix C describes the asymptotic properties of combined censoring and sleeping when the SNR approaches infinity. Note that this analysis is preformed merely in order to gain analytical insight in to the proposed scheme at extreme values of the SNR. In practice such a situation is possible when the cognitive radios 
are either in the close range of the primary user, or there is a line of sight (LOS) channel to the primary user.

Theorem 3 If $\forall j: \gamma_{j} \rightarrow \infty$, the optimal solution to (31) and (50) is obtained by the maximum sleeping rate $\mu$, in the feasible set of (31) and (50), respectively.

Therefore, when the SNR is high, using Theorem 3, a suboptimal solution can be obtained which is close to the optimal solution.

\section{Some Notes on the Deterministic Scenario}

So far, we have focused on the two models which are described under (1) in Section III, where the received samples at each sensor are generated by an i.i.d. Gaussian random process with zero-mean under $\mathcal{H}_{0}$ and $\mathcal{H}_{1}$. In this section, we assume a model where the channel is time-invariant, i.e., $\forall i: h_{i, j}=h_{j}$ and the signal is deterministic, i.e., $\left|s_{i}\right|=s$. In this case, the received samples at the sensors are not zeromean anymore under $\mathcal{H}_{1}$. This case can be considered as an unknown deterministic signal detection scenario such as the one in [37], employing an energy detector. This scenario represents a case where symbol duration is comparable to channel coherence time [31]. The received SNR at the $j$-th cognitive radio in this case is $\gamma_{j}=\left|h_{j}\right|^{2}|s|^{2} / \sigma_{w}^{2}$.

Employing the energy detector in (2), $\mathcal{E}_{j}$ follows a chisquare distribution with $2 N$ degrees of freedom under $\mathcal{H}_{0}$ and a non-central chi-square distribution with $2 N$ degrees of freedom and non-centrality parameter $\gamma_{j}$ under $\mathcal{H}_{1}$. This way, the local probabilities of false alarm and detection at the $j$-th cognitive sensor, denoted by $P_{f, d e t, j}$ and $P_{d, d e t, j}$, respectively, are obtained as follows

$$
\begin{gathered}
P_{f, \text { det }, j}=\operatorname{Pr}\left(\mathcal{E}_{j} \geq \lambda_{2} \mid \mathcal{H}_{0}\right)=\frac{\Gamma\left(N, \frac{\lambda_{2}}{2}\right)}{\Gamma(N)}, \\
P_{d, \text { det }, j}=\operatorname{Pr}\left(\mathcal{E}_{j} \geq \lambda_{2} \mid \mathcal{H}_{1}\right)=Q_{2 N}\left(\sqrt{2 \gamma_{j}}, \sqrt{\lambda_{2}}\right),
\end{gathered}
$$

where $Q_{u}(c, x)$ is the generalized Marcum Q-function, $Q_{u}(c, x)=\frac{1}{c^{u-1}} \int_{x}^{\infty} t^{u} e^{-\frac{t^{2}+c^{2}}{2}} I_{u-1}(c t) d t$, with $I_{u-1}($.$) be-$ ing the modified Bessel function of the first kind and order $u-1$. The rest of the analysis, which is discussed in Section IV, is independent from the type of samples which are received at the sensors, as long as they are conditionally independent. Therefore, in order to solve (14) and (39) for the deterministic scenario, we only need to substitute (52) and (53) instead of (4) and (5) in the related equations. Note that here, the exact solution to the underlying problems is not necessarily the sames as for random signals, but the algorithms to solve the problems are similar.

\section{NUMERICAL RESULTS}

In this section, we first analyze the performance of the system numerically by assuming some arbitrary values for the sensing and transmission energy in Section VI-A. A case study is then discussed in order to evaluate the energy-efficiency of the proposed scheme in Section VI-B.

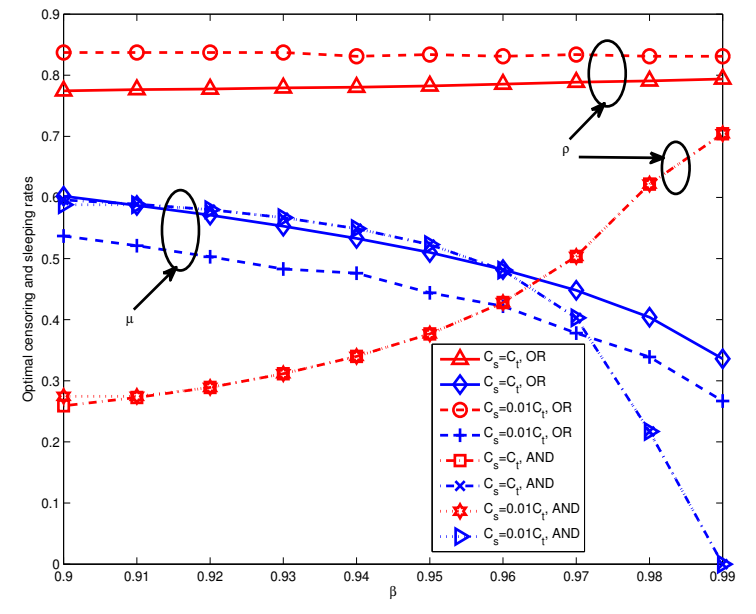

Fig. 2. Optimal sleeping and censoring rate for $\alpha=0.1$ and $0.9 \leq \beta \leq 0.99$

\section{A. Numerical Analysis}

A network of five cognitive radios is considered. Each cognitive radio receives the primary user signal with an average SNR of $\gamma=10 \mathrm{~dB}$ and the number of observation samples is equal to $N=5$. Here, the goal is to analyze the behavior of the optimal censoring and sleeping rate as the detection performance constraint of the system changes. Further in Figs. 2 and 3, in one scenario, the sensing and transmission energy is assumed to be the same and in the other one, the transmission energy is assumed to be 100 times larger than the sensing energy.

The optimal censoring and sleeping rates are depicted in Fig. 2 for different values of $\beta$ and $\alpha=0.1$. We can see that as the transmission energy increases with respect to the sensing energy, the censoring rate increases while the sleeping rate decreases for the OR rule. The reason is that as the transmission energy becomes larger than the sensing energy, the combined censoring and sleeping scheme tends to save more energy on transmission compared to the one on sensing. Although the same trend can be observed for the AND rule, the optimal censoring and sleeping rates for the AND rule are approximately the same for the different sensing and transmission energies. As discussed in Section IV-C, this behavior comes from the fact that at high SNRs, the optimal parameters of the underlying problem become independent from the values of the sensing and transmission energies. Further, it seems this behavior appears at lower SNRs for the AND rule with respect to the OR rule.

The same behavior as in Fig. 2 is shown in Fig. 3, where the optimal censoring and sleeping rates for $0.03 \leq \alpha \leq 0.1$ and $\beta=0.9$ are depicted. Similar to Fig 2, we can see that as the transmission energy increases with respect to the sensing energy, the optimal censoring rate increases while the sleeping rate decreases. Again we can see that due to the high SNR, for the AND rule, the optimal censoring and sleeping rates are approximately the same for different values of the sensing and transmission energies.

In Fig. 4, the optimal average energy consumption per 


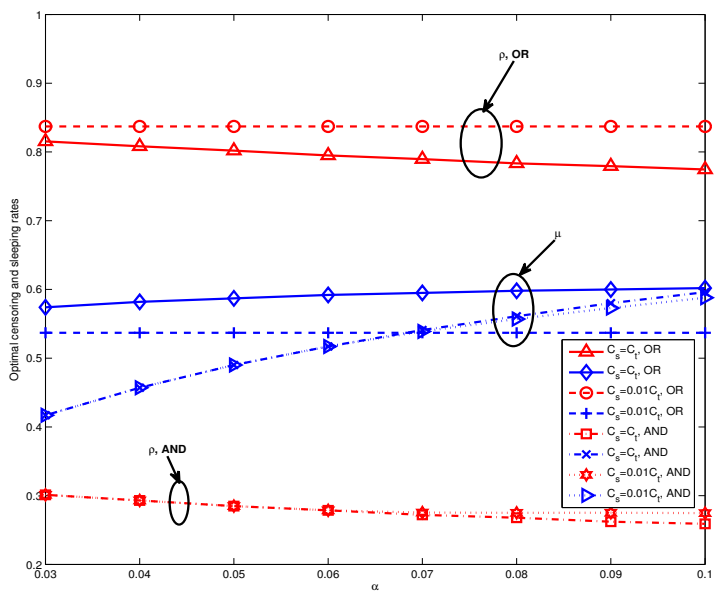

Fig. 3. Optimal sleeping and censoring rate for $0.03 \leq \alpha \leq 0.1$ and $\beta=0.9$

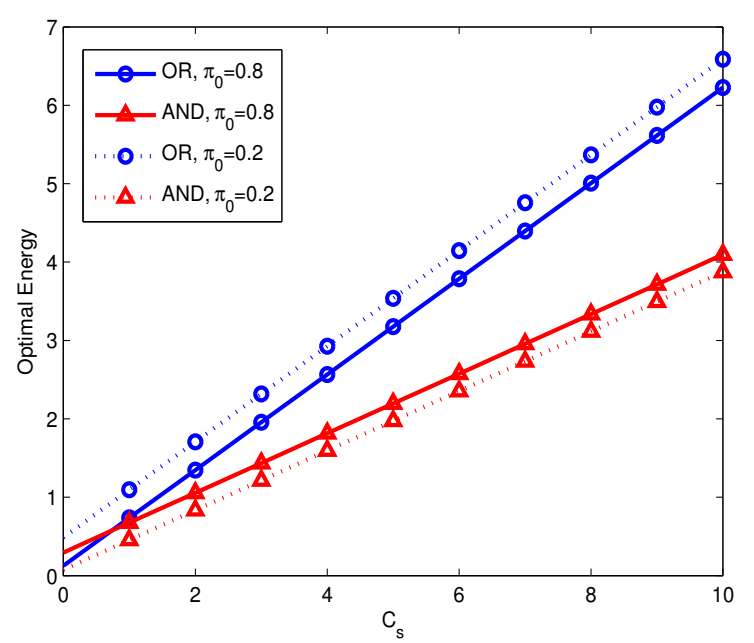

Fig. 4. Optimal average energy consumption per sensor versus the sensing energy with $\alpha=0.1, \beta=0.99, C_{t}=1$. The AND rule outperforms the OR rule after a specific $C_{s}$.

sensor is depicted versus the sensing energy $C_{s}$ for $\pi_{0}=0.2$ and $\pi_{0}=0.8$, in order to compare the performance of the OR and the AND rule. In this figure, $M=5, N=5, \gamma=10 \mathrm{~dB}$, $\alpha=0.1$ and $\beta=0.99, C_{t}=1$ and $C_{s}$ changes from 0 to 10 . We can see that the AND rule outperforms the OR rule as $C_{s}$ increases. Therefore, for the desired constraints on the probability of detection and false alarm in this figure, the AND rule seems a better choice compared to the OR rule, particularly when the sensing energy is much higher with respect to the transmission energy.

\section{B. Case Study for IEEE 802.15.4/ZigBee}

We consider a case study based on IEEE 802.15.4/ZigBee in order to evaluate the performance of combined sleeping and censoring. A circular network of $M$ cognitive radios is considered where cognitive radios are uniformly distributed around the circle and the FC is located at the center. The radius of the circle and thus the distance between each cognitive radio and the $\mathrm{FC}$ is assumed to be $70 \mathrm{~m}$. Each cognitive radio is a Chipcon CC2420 transceiver which operates according to the IEEE 802.15.4/ZigBee standard [38]. The sensing and transmission energy is calculated based on the specification of this transceiver. A free-space path loss model is employed to model the wireless channel between the cognitive radio and the FC and this leads to a signal attenuation which is inversely proportional to the square of the distance $d$ between the transmitter and receiver. The number of samples is assumed to be $N=5$, which corresponds to a sensing time of $1 \mu \mathrm{s}$. The total sensing energy consisting of listening and processing energy for 5 samples is derived in [20] and is roughly equal to $5 C_{s}=190 \mathrm{~nJ}$. Following the same model as in [20], the transmission energy for a range of $70 \mathrm{~m}$ and transmission of a one-bit decision, is approximately $C_{t}=278 \mathrm{~nJ}$. Note that the transmission energy is derived in order to satisfy a receiver sensitivity of $-90 \mathrm{dBm}$ at a SNR of $10 \mathrm{~dB}$.

Fig. 5 depicts the optimal average energy consumption per sensor versus the probability of detection constraint, $\beta$. The number of cognitive radios is $M=5$, the probability of false alarm constraint $\alpha=0.1$ and $0.9 \leq \beta \leq 0.99$. We let $\pi_{0}$ to adopt three values including $\{0.2,0.8,1\}$ reflecting the cases where the probability of primary user absence is low, high, and extremely high, respectively. We can see that the combined censoring and sleeping scheme delivers a high energy saving compared to the scenario where no energy-efficient scheme is considered. We further notice that the AND rule outperforms the OR rule for low values of $\pi_{0}$ reflecting the lower chance of reporting a 0 and thus a higher censoring rate compared to the OR rule as shown in Fig. 6. The opposite trend is shown for the case where $\pi_{0}$ is high. For example, except for the high values of $\beta$, the OR rule outperforms the AND rule when $\pi_{0}=0.8$. For the extremely high values of $\pi_{0}$, it is shown that the OR rule always outperforms the AND rule with much higher censoring rate as shown in Fig. 6. It is also shown that in accordance to Corollary 1 the lower-bound on the optimal average energy consumption per sensor for the OR rule is achieved when $\pi_{0}=1$. Further, Theorems 1 and 2 are also verified in Fig. 5. The optimal average energy consumption per sensor increases with $\pi_{0}$ for the AND rule and decreases with $\pi_{0}$ for the OR rule.

Fig. 6 shows the optimal censoring and sleeping rate versus the probability of detection constraint $\beta$ for the same parameters as in Fig. 5. This figure helps us in understanding the observed behaviors in Fig. 5. It is shown that under the considered parameters in this scenario, due to the high SNR, the sleeping rate has the same value for different a priori probabilities $\pi_{0}$ and $\pi_{1}$ as well as for the blind setup. This behavior verifies the discussion in Section IV-C. Further, we can see that the censoring rate increases with $\pi_{0}$ and is the largest for $\pi_{0}=1$ for the OR rule and that is why the blind setup (which assumes $\pi_{0}>>\pi_{1}$ ) gives the lowerbound on the energy-efficiency of the combined censoring and sleeping scheme. The opposite behavior can be observed for the AND rule as $\pi_{0}$ increases. Note that here and in the rest of this section, our goal is not to compare the performance of the knowledge-aided and blind setups, but to just show 


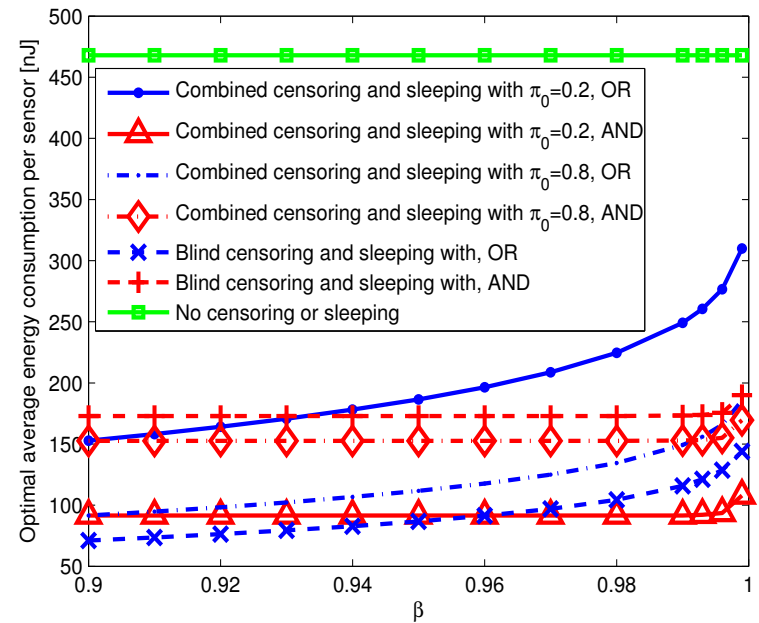

Fig. 5. Comparison of energy consumption versus the probability of detection constraint. Significant energy savings can be achieved.

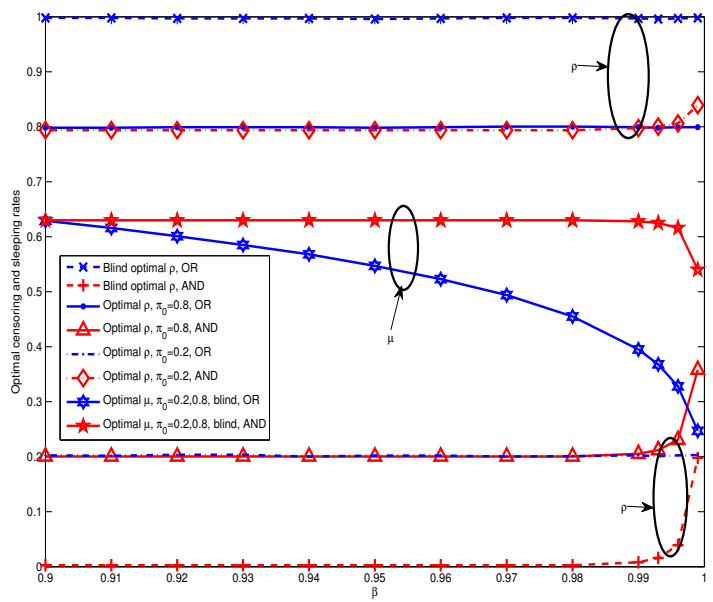

Fig. 6. Optimal censoring and sleeping rate versus the probability of detection constraint

the expected performance of the proposed algorithm under each setup. When we refer to the blind setup, we intend to study the performance of combined censoring and sleeping asymptotically as $\pi_{0} \rightarrow 1$.

The optimal average energy consumption per sensor is drawn in Fig. 7 versus the number of cognitive radios. In this figure, the global probabilities of false alarm and detection are assumed to be $\alpha=0.1$ and $\beta=0.9$. Again it is shown that combined censoring and sleeping is very promising in terms of energy-efficiency with respect to the scenarios where no energy-efficiency is taken into account. We can see that as the number of cognitive radios increases, the system gains a higher energy saving, reflecting a lower burden on the individual cognitive radios. As in Fig. 5, the AND rule outperforms the OR rule in low values of $\pi_{0}$, while the OR rule outperforms the AND rule for high values of $\pi_{0}$. We can see again that the lower-bound on the optimal average

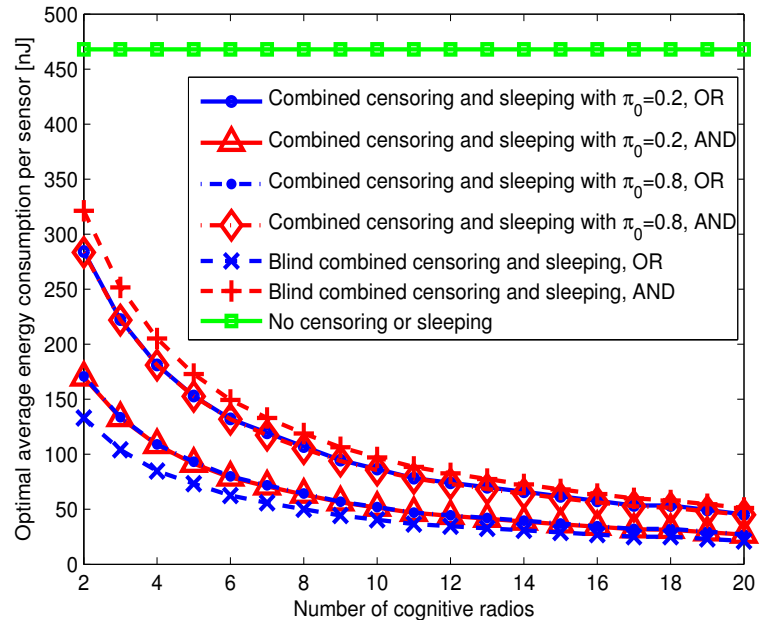

Fig. 7. Energy scaling with number of cognitive sensors for different setups. Increasing the number of cognitive radios reduces the energy burden of individual cognitive radios.

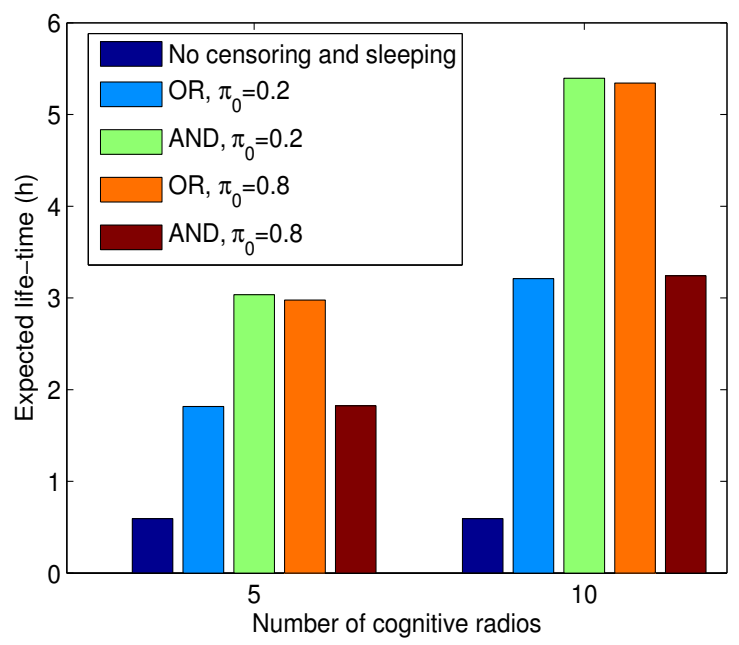

Fig. 8. Expected life-time of the sensors, with $\mathcal{C}_{T}=1000 \mathrm{~J}, T_{s}=1 \mu \mathrm{s}$, $\alpha=0.1, \beta=0.9$

energy consumption per sensor for the OR rule is achieved when $\pi_{0}=1$. Figures 5 and 7 show that the average energy consumption per sensor in each sensing slot can be reduced significantly which in turn increases the expected life-time of the sensors. Denoting the expected life-time of a sensor by $\mathcal{L}_{j}$, the total available energy for sensing by $\mathcal{C}_{T}$, and the sensing duration by $T_{s}$, the expected life-time of the sensor is obtained by $\mathcal{L}_{j}=T_{s} \frac{\mathcal{C}_{T}}{C_{j}}$. The fact that the life-time of the sensors increases by employing combined censoring and sleeping is depicted in Fig. 8. In this figure, $\mathcal{C}_{T}=1000 \mathrm{~J}$, and the remaining parameters are the same as Fig. 7. Here, again we can see that increasing the number of cognitive radios can improve the life-time of individual sensors significantly.

The optimal censoring and sleeping rates versus the number of users is depicted in Fig. 9 in order to understand the observed behaviors in Fig. 7. The results are obtained for 


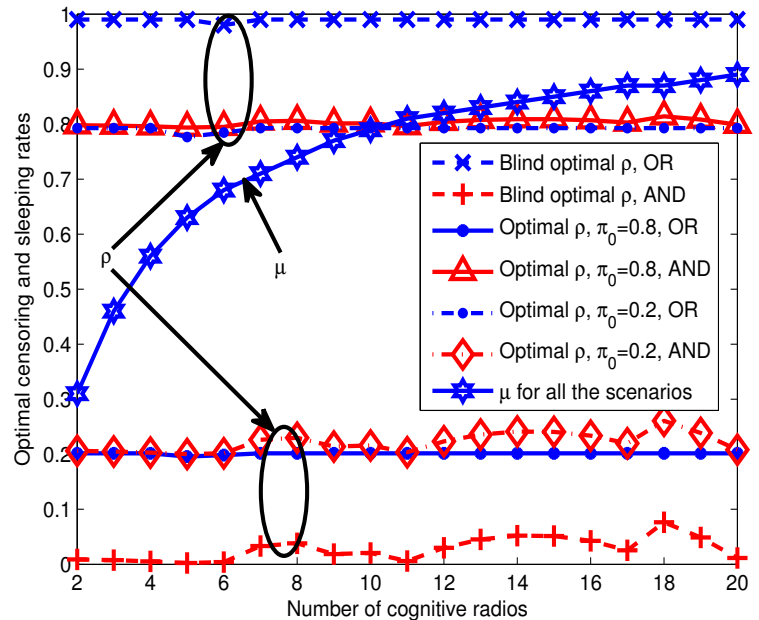

n

Fig. 9. Optimal censoring and sleeping rate with number of cognitive radios

the same setup as the one in Fig. 7. We can see that as the number of users increases, the optimal sleeping rate increases in order to reduce the average energy consumption per sensor. However, the optimal censoring rate remains almost the same. Again considering the discussions in Section IV-C, we can see that due to a high SNR, the optimal sleeping rate becomes independent from $\pi_{0}$ and that is why the sleeping rate among all the scenarios remains the same. Further, we can see that as $\pi_{0}$ increases, the censoring rate increases for the OR rule and decreases for the AND rule which results in a lower average energy consumption per sensor for the OR rule and a higher one for the AND rule. This verifies the validity of Theorems 1 and 2. A specific duality is also observed between the OR and the AND rule in this figure. While the optimal sleeping rate is the same for both, the optimal censoring rate for a specific $\pi_{0}$ and the OR rule is approximately the same as for the AND rule for a probability of the primary user absence with a value of $1-\pi_{0}$. This property which can also be seen in Fig. 7 is investigated more in the following scenarios.

In all of the following scenarios, the number of cognitive radios is $M=5$, the number of samples is $N=5$ and the SNR is $\gamma=10 \mathrm{~dB}$.

In Figs. 10, 11 and 12, the optimal average energy consumption per sensor is depicted versus the probability of primary user absence, $\pi_{0}$. The goal is to study performance of the proposed algorithm for the OR and the AND rules when $\pi_{0}$ as well as the detection constraints change. In these figures, the probability of false alarm constraint $\alpha=0.1$, and the probability of detection constraint $\beta=0.8,0.9$, and 0.99 respectively, in Figs. 10, 11 and 12. We can see that as $\pi_{0}$ increases, the average energy consumption per sensor reduces for the OR rule, while for the AND rule, it increases, which is in line with Theorems 1 and 2. Intuitively speaking, in the lower values of $\pi_{0}$ for the OR rule, on average, a higher number of transmissions occurs compared to the higher values of $\pi_{0}$, because the FC in the case of the OR rule only receives 1s from the users. In contrast to the OR rule, for the AND rule, the probability that cognitive users transmit their results to the FC increases by increasing $\pi_{0}$, since the probability of sending $0 \mathrm{~s}$ to the FC increases. Therefore, the average energy consumption per sensor decreases and increases with $\pi_{0}$, for the OR and AND rules, respectively.

Moreover, in Fig. 11, an interesting behavior in the optimal average energy consumption per sensor is shown with $\pi_{0}$. We can see that for $\pi_{0}<0.5$, the AND rule outperforms the OR rule, while for $\pi_{0}>0.5$, it is vice versa, and for $\pi_{0}=0.5$, both rules almost behave the same. The same behavior can be shown to appear when $\alpha+\beta=1$. This duality between the OR and the AND rules is observed in Figs. 7 and 9. We can see in Figs. 10 and 12, that with decreasing or increasing $\beta$, the crossing point where the OR rule starts to outperform the AND rule moves respectively to the left or the right of $\pi_{0}=0.5$. Similar to $\beta$, we can show that increasing or decreasing $\alpha$ with a constant $\beta$ moves the performance crossing point of the OR and the AND rule to the right or left, respectively.

\section{CONCLuSions AND Future Works}

A combined censoring and sleeping scheme was discussed as an energy-efficient distributed spectrum sensing technique in cognitive radio networks. The underlying parameters including the lower threshold $\lambda_{1}$, the upper threshold $\lambda_{2}$, and the sleeping rate $\mu$ were obtained by minimizing the maximum average energy consumption per sensor subject to a specific detection performance for the OR and the AND rule. It was shown that the optimal $\lambda_{1}$ is $\lambda_{1}=0$ for the OR and $\lambda_{1} \rightarrow \infty$ for the AND rule. Employing the interaction between $\lambda_{2}$ and $\mu$, we showed that the problem can be solved by a line search over $\mu$ for both the OR and the AND rule. Further, we showed that the same solutions can be employed to solve the problem for a deterministic scenario, where the primary user signal is deterministic and the channel is time-invariant. Asymptotic properties of the OR and the AND rule were discussed as $\pi_{0} \rightarrow 0$ or 1 , and also when the SNR approaches infinity. It was shown that the optimal average energy consumption per sensor is increasing with $\pi_{0}$ for the AND rule and decreasing with $\pi_{0}$ for the OR rule. This way the lower-bound and upper-bound on the energy consumption per sensor is obtained respectively for the $\mathrm{OR}$ and the AND rule when $\pi_{0}=1$. It was depicted that as the SNR approaches infinity, the optimal solution of the underlying problems becomes independent from the value of $\pi_{0}$ as well as the values of the sensing and transmission energies.

Several scenarios with different values of the sensing and transmission energy as well as different probabilities of false alarm and detection constraints were considered in the numerical results. It was shown that as the transmission energy increases with respect to the sensing energy, the optimal censoring rate increases, while the optimal sleeping rate decreases. A case study based on the IEEE 802.15.4/ZigBee standard was considered where we showed that a significant energy saving can be achieved by combined sleeping and censoring. It was shown that for the desired detection performance of a cognitive radio system defined by $\alpha=0.1$ and $\beta=0.9$, the OR rule outperforms the AND rule for $\pi_{0}>0.5$, while the AND rule outperforms the OR rule when $\pi_{0}<0.5$. 


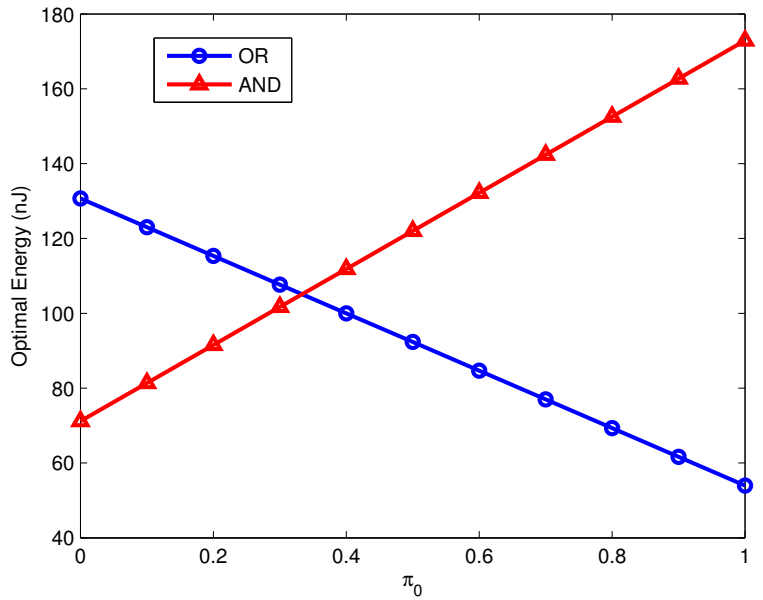

Fig. 10. Optimal average energy consumption per sensor versus the probability of primary user absence for $\alpha=0.1$ and $\beta=0.8$

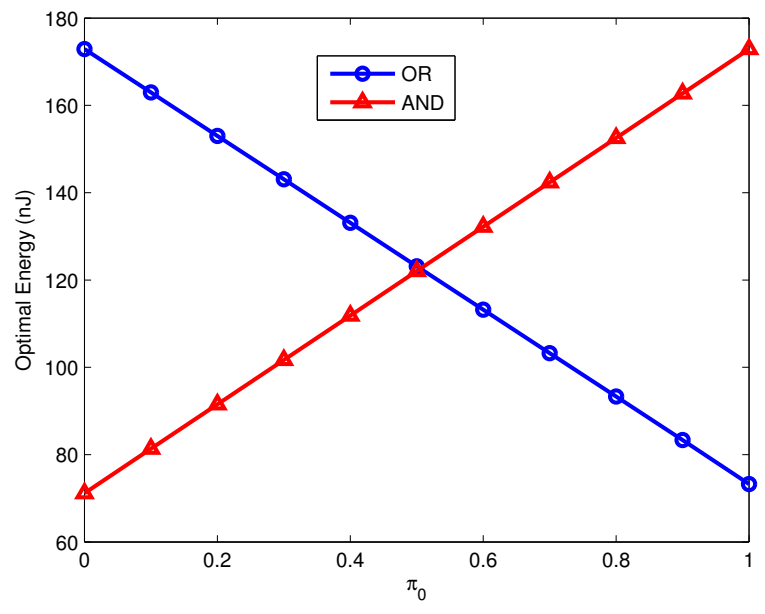

Fig. 11. Optimal average energy consumption per sensor versus the probability of primary user absence for $\alpha=0.1$ and $\beta=0.9$.

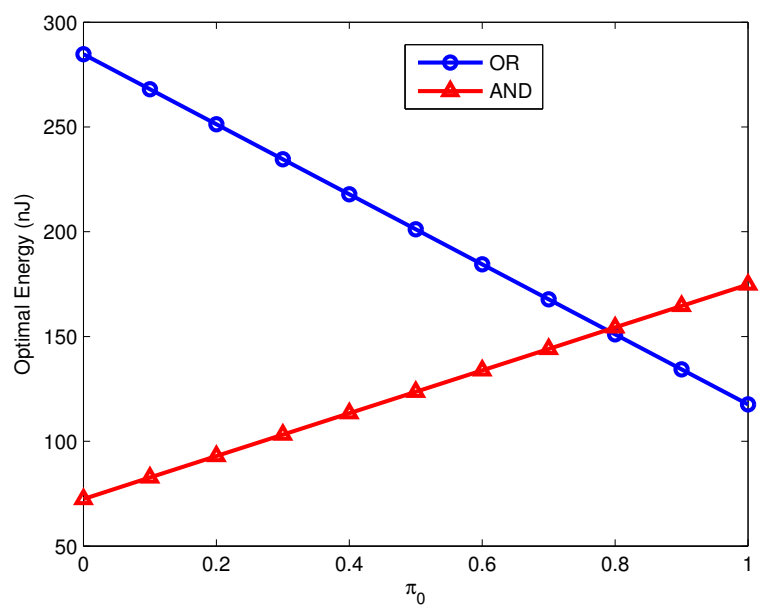

Fig. 12. Optimal average energy consumption per sensor versus the probability of primary user absence for $\alpha=0.1$ and $\beta=0.99$

It was shown that increasing the number of cognitive users, not only improves the detection reliability of the cognitive radio network but also, if the system is well-designed in terms of energy-efficiency, reduces the burden on the individual cognitive radios in terms of energy consumption.

Note that in this paper, we considered a distributed spectrum sensing scheme with a fusion center. There are cases though where a fusion center is not available, in which case decentralized distributed spectrum sensing is required. To the best of our knowledge, energy-efficient decentralized distributed spectrum sensing is not considered in the literature. Employing selective transmission schemes such as the ones in [39] and [40] to design energy-efficient algorithms for decentralized distributed sensing is a subject of further study.

Here, we only considered the combined censoring and sleeping scheme for the OR and the AND rule. Extension of the scheme to more general hard fusion rules is another subject for future work. In this paper, we solved the final reduced one-dimensional problems by exhaustive search over the sleeping rate. Designing efficient algorithmic solutions to solve these problems are also valuable ideas for future studies.

\section{APPENDIX A \\ ProOF OF THEOREM 1}

Imagine $\mu^{*}$ is the solution to (31). Since $\forall \mu, \beta$ in the feasible set of (31), $F\left(G^{-1}(\mu, \beta) /\left(1+\gamma_{j}\right)\right) \geq F\left(G^{-1}(\mu, \beta)\right)$, we obtain $C_{j}\left(\pi_{0}^{\prime}, \mu^{*}\right) \leq C_{j}\left(\pi_{0}, \mu^{*}\right)$ if $\pi_{0}^{\prime}>\pi_{0}$. Note that equality is achieved when $\gamma_{j} \rightarrow 0$. Further, assume that $\mu^{*^{\prime}}$ is the optimal solution to (31) for $\pi_{0}^{\prime}$. Then we know that $C_{j}\left(\pi_{0}^{\prime}, \mu^{*^{\prime}}\right) \leq C_{j}\left(\pi_{0}^{\prime}, \mu^{*}\right)$ and thus $C_{j}\left(\pi_{0}^{\prime}, \mu^{*^{\prime}}\right) \leq C_{j}\left(\pi_{0}, \mu^{*}\right)$ which proves Theorem 1 .

\section{APPENDIX B \\ PROOF OF THEOREM 2}

Imagine $\mu^{*}$ is the optimal solution to (50). Since $\forall \mu, \alpha$ in the feasible set of (50), $\delta_{1, j} \geq \delta_{0}$, therefore, we have $F_{j, \mathrm{AND}}(1-$ $\left.\frac{1-\alpha^{1 / M}}{1-\mu}\right) \geq \frac{1-\alpha^{1 / M}}{1-\mu}$. This way, we obtain $C_{j}\left(\pi_{0}^{\prime}, \mu^{*}\right) \leq$ $C_{j}\left(\pi_{0}, \mu^{*}\right)$ if $\pi_{0}^{\prime}<\pi_{0}$. Note that equality is achieved when $\gamma_{j} \rightarrow 0$. Further, assume that $\mu^{*^{\prime}}$ is the optimal solution to (50) for $\pi_{0}^{\prime}$. Then we know that $C_{j}\left(\pi_{0}^{\prime}, \mu^{*^{\prime}}\right) \leq C_{j}\left(\pi_{0}^{\prime}, \mu^{*}\right)$, and thus $C_{j}^{*^{\prime}} \leq C_{j}^{*}$ which proves Theorem $2 . \square$

\section{APPENDIX C \\ PROOF OF THEOREM 3}

First we note that when $\forall j: \gamma_{j} \rightarrow \infty$, the local probabilities of detection become very close to each other and thus we let $P_{d, j}=P_{d}$ in (18). The same happens for $\delta_{1, j}$ in (41) and hence we also let $\delta_{1, j}=\delta_{1}$. This way $Q_{\mathrm{D}, \mathrm{OR}}=1-\left[1-(1-\mu) P_{s}\right]^{M}$ and $Q_{\mathrm{D}, \mathrm{AND}}=\left[1-(1-\mu)\left(1-\delta_{1}\right)\right]^{M}$.

From (28) and (30), we can see that for a given $\mu$, the optimal $P_{d}$ is equal to $\frac{1-(1-\beta)^{1 / M}}{1-\mu}$. This way, (31), becomes $\min _{\mu}(1-\mu)\left[N C_{s}+C_{t}\left(\pi_{0} F\left((1+\gamma) F^{-1}\left(\frac{1-(1-\beta)^{1 / M}}{1-\mu}\right)\right)+\right.\right.$ $\left.\left.\pi_{1} \frac{1-(1-\beta)^{1 / M}}{1-\mu}\right)\right]$. We note that when the SNR approaches infinity, the optimal $P_{f}=F\left((1+\gamma) F^{-1}\left(\frac{1-(1-\beta)^{1 / M}}{1-\mu}\right)\right)$ 
approaches 0 and thus (31) reduces to $\min _{\mu}\left[(1-\mu) N C_{s}+\right.$ $\left.\pi_{1} C_{t}\left(1-(1-\beta)^{1 / M}\right)\right]$, and this problem is solved by finding the maximum $\mu$ in the feasible set of (31).

Further, when the SNR approaches infinity, the optimal $\delta_{1}$ approaches 1 , since $F\left(F^{-1}\left(\delta_{0}\right) /(1+\gamma)\right) \rightarrow 1$. This way, (50) reduces to $\min _{\mu}\left[(1-\mu) N C_{s}-\pi_{0} C_{t}\left(1-\alpha^{1 / M}\right)\right]$, which is solved again by finding the maximum $\mu$ in the feasible set of (50) which is $\mu=\alpha^{1 / M}$.

\section{REFERENCES}

[1] R. W. Broderson, A. Wolisz, D. Cabric, S. M. Mishra and D. Willkomm. White paper: CORVUS: A Cognitive Radio Approach for Usage of Virtual Unlicensed Spectrum. Technical report, 2004.

[2] Q. Zhao and B. M. Sadler, "A Survey of Dynamic Spectrum Access," IEEE Signal Processing Magazine, pp 79-89, May 2007.

[3] A. Goldsmith, S. A. Jafar, I. Maric and S. Srinivasa, "Breaking Spectrum Gridlock With Cognitive Radios: An Information Theoretic Perspective," Proceedings of the IEEE, vol.97, no.5, pp.894,914, May 2009.

[4] D. Cabric, S. M. Mishra and R. W. Brodersen, "Implementation issues in spectrum sensing for cognitive radios," Asilomar Conference on Signals, Systems and Computers, pp 772-776, Nov. 2004.

[5] R. Tandra and A. Sahai, "SNR walls for signal detection", IEEE Journal of Selected Topics in Signal Processing, pp. 4-17, Feb. 2008.

[6] E. Axell, G. Leus, E. G. Larsson and H. V. Poor, "Spectrum Sensing for Cognitive Radio : State-of-the-Art and Recent Advances," IEEE Signal Processing Magazine, vol.29, no.3, pp.101,116, May 2012.

[7] C. R. C. da Silva, B. Choi and K. Kim, "Cooperative Sensing among Cognitive Radios," Information Theory and Applications Workshop, pp 120-123, 2007.

[8] S. M. Mishra, A. Sahai and R. W. Brodersen, "Cooperative Sensing among Cognitive Radios," IEEE International Conference on Соттиnications, pp 1658-1663, June 2006.

[9] P. K. Varshney, Distributed Detection and Data Fusion, Springer, 1996.

[10] S. Appadwedula, V. V. Veeravalli and D. L. Jones, "Decentralized Detection With Censoring Sensors," IEEE Transactions on Signal Processing, pp 1362-1373, Apr. 2008.

[11] S. Appadwedula, V. V. Veeravalli and D. L. Jones, "Energy-efficient detection in sensor networks," IEEE Journal on Selected Areas in Communications, pp 693-702, Apr. 2005.

[12] C. Rago, P. Willett and Y. Bar-Shalom, "Censoring sensors: a lowcommunication-rate scheme for distributed detection," IEEE Transactions on Aerospace and Electronic Systems, pp 554-568, Apr. 1996.

[13] C. Sun, W. Zhang and K. B. Letaief, "Cooperative Spectrum Sensing for Cognitive Radios under Bandwidth Constraints," IEEE Wireless Communications and Networking Conference, March 2007.

[14] Y. Chen, "Analytical Performance of Collaborative Spectrum Sensing Using Censored Energy Detection," IEEE Transactions on Wireless Communications, vol.9, no.12, pp.3856,3865, December 2010.

[15] S. Maleki and G. Leus, "Censored Truncated Sequential Spectrum Sensing for Cognitive Radio Networks," IEEE Journal on Selected Areas in Communications, vol.31, no.3, pp.364,378, March 2013.

[16] J. Lunden, V. Koivunen, A. Huttunen and H. V. Poor, "Collaborative Cyclostationary Spectrum Sensing for Cognitive Radio Systems," IEEE Transactions on Signal Processing, vol.57, no.11, pp.4182,4195, Nov. 2009.

[17] A. V. Dandawate, G. B. Giannakis, "Statistical tests for presence of cyclostationarity," IEEE Transactions on Signal Processing, vol.42, no.9, pp.2355,2369, Sep. 1994

[18] K. Yamasaki and T. Ohtsuki, "Design of energy-efficient wireless sensor networks with censoring, on-off, and censoring and on-off sensors based on mutual information," IEEE Vehicular Technology Conference, pp 13121316, 2005.

[19] D. Datla, R. Rajbanshi, A. M. Wyglinski and G. J. Minden, "An adaptive spectrum sensing architecture for dynamic spectrum access networks," IEEE Transactions on Wireless Communications, Aug. 2009.

[20] S. Maleki, A. Pandharipande and G. Leus, "Energy-Efficient Distributed Spectrum Sensing for Cognitive Sensor Networks," IEEE Sensors Journal, vol.11, no.3, pp.565,573, March 2011.
[21] M. Najimi, A. Ebrahimzadeh, S. M. H. Andargoli and A. Fallahi, "A Novel Sensing Nodes and Decision Node Selection Method for Energy Efficiency of Cooperative Spectrum Sensing in Cognitive Sensor Networks," IEEE Sensors Journal, vol.13, no.5, pp.1610,1621, May 2013.

[22] C.-H. Lee and W. Wolf, "Energy Efficient Techniques for Cooperative Spectrum Sensing in Cognitive Radios," IEEE Consumer Communications and Networking Conference, pp 968-972, Jan. 2008.

[23] Y. Chen, J. A. Nossek and A. Mezghani, "Circuit-Aware Cognitive Radios for Energy-Efficient Communications," IEEE Wireless Communications Letters, vol.2, no.3, pp.323,326, June 2013.

[24] S. Maleki, S. P. Chepuri and G. Leus, "Optimization of hard fusion based spectrum sensing for energy-constrained cognitive radio networks", Physical Communication, Available online 20 July 2012.

[25] E. C. Y. Peh, Y. C. Liang, Y. L. Guan and Y. Pei, "Energy-Efficient Cooperative Spectrum Sensing in Cognitive Radio Networks," 2011 IEEE Global Telecommunications Conference (GLOBECOM 2011), vol., no., pp.1,5, 5-9 Dec. 2011.

[26] Y. Pei, Y. C. Liang, K. C. Teh and K. H. Li, "Energy-Efficient Design of Sequential Channel Sensing in Cognitive Radio Networks: Optimal Sensing Strategy, Power Allocation, and Sensing Order," IEEE Journal on Selected Areas in Communications, vol.29, no.8, pp.1648,1659, September 2011.

[27] S. Wang, Y. Wang, J. P. Coon and A. Doufexi, "Energy-Efficient Spectrum Sensing and Access for Cognitive Radio Networks," IEEE Transactions on Vehicular Technology, vol.61, no.2, pp.906,912, Feb. 2012.

[28] J. A. Han, W. S. Jeon and D. G. Jeong, "Energy-Efficient Channel Management Scheme for Cognitive Radio Sensor Networks," IEEE Transactions on Vehicular Technology, vol.60, no.4, pp.1905,1910, May 2011.

[29] H. Ehm, S. Winter and R. Weigel, "Analytic quantization modeling of OFDM signals using normal Gaussian distribution," Asia-Pacific Microwave Conference, 2006. APMC 2006, pp.847,850, 12-15 Dec. 2006.

[30] C. Phillips, M. Ton, D. Sicker, and D. Grunwald, "Practical radio environment mapping with geostatistics," 2012 IEEE International Symposium on Dynamic Spectrum Access Networks (DYSPAN), pp.422,433, 16-19 Oct. 2012.

[31] S. K. Sharma, S. Chatzinotas and B. Ottersten, "Eigenvalue Based Sensing and SNR Estimation for Cognitive Radio in Presence of Noise Correlation," IEEE Transactions on Vehicular Technology, vol.62, no.8, pp.3671,3684, Oct. 2013.

[32] D. Cabric, A. Tkachenko and R. W. Brodersen, "Experimental study of spectrum sensing based on energy detection and network cooperation," First international workshop on Technology and policy for accessing spectrum, 2006.

[33] D. Cabric, A. Tkachenko and R. W. Brodersen, "Spectrum Sensing Measurements of Pilot, Energy, and Collaborative Detection," IEEE Military Communications Conference, 2006. MILCOM 2006., pp.1,7, 2325 Oct. 2006.

[34] R. Deng, J. Chen, C. Yuen, P. Cheng and Y. Sun, "Energy-Efficient Cooperative Spectrum Sensing by Optimal Scheduling in Sensor-Aided Cognitive Radio Networks," IEEE Transactions on Vehicular Technology, vol.61, no.2, pp.716,725, Feb. 2012.

[35] S. M. Kay, Fundamentals of Statistical Signal Processing, Volume 2: Detection Theory, Prentice Hall, 1998.

[36] S. Boyd and L. Vandenberghe, Convex Optimization, Cambridge University Press, 2004

[37] H. Urkowitz, "Energy detection of unknown deterministic signals," Proceedings of the IEEE, vol.55, no.4, pp.523,531, April 1967.

[38] IEEE 802.15.4 standard, Part 15.4: Wireless Medium Access Control (MAC) and Physical Layer (PHY) Specifications for Low-Rate Wireless Personal Area Networks (WPANs), 2006.

[39] R. Arroyo-Valles, A. G. Marques and J. Cid-Sueiro, "Optimal Selective Transmission under Energy Constraints in Sensor Networks," IEEE Transactions on Mobile Computing, vol.8, no.11, pp.1524,1538, Nov. 2009.

[40] R. Arroyo-Valles, S. Maleki and G. Leus, "A Censoring Strategy for Decentralized Estimation in Energy-Constrained Adaptive Diffusion Networks", The 14th IEEE International Workshop on Signal Processing Advances in Wireless Communications, SPAWC 2013, pp. 146-150, June 2013.

[41] S. Maleki, G. Leus, S. Chatzinotas and B. Ottersten, "To AND or To OR: How Shall the Fusion Center Rule in Energy-Constrained Cognitive Radio Networks?", 2014 IEEE International Conference on Communications, ICC 2014, pp. 1632-1637, June 2014. 